\title{
STRUCTURE AND FUNCTIONS
}

or

\section{THE H U A N PLA CENTA.}

\author{
By J OH N DARYMPLE, EsQ.,
}

AsEISTANT-SURGEON TO THE OPHTHALMIC INPIRMARY, MOORFIELDS.

READ NOVEMBER 23RD, 1841.

The views submitted to the consideration of the Society in the present brief account of the structure of the human placenta, are principally corroborative of the description of Weber, and the summary given in the first number of R. Wagner's Physiology, as translated by Dr. Willis. The drawings which accompany this paper were made without reference to the plates of that work, and from actual specimens of the injected organ: yet so closely do they agree with the copies of the Icones Physiologicæ given by Dr. Willis, as to almost induce the belief of their being mere imitations. This very correspondence, however, while it deprives me of the claim to originality, justifies me in offering the investigations of this structure, as confirmatory of the views of the accurate and laborious foreigner.

So much has been written upon the placenta, and 
so various and discrepant have been the descriptions given of its anatomy, and so multiform the theories of its physiology, that $I$ have been induced to propose a simplification both of its physical structure and uses.

It will be shown, in the first place, that any direct communication between the uterine vessels and those of the placenta is impossible, and that, though it is not denied that injections may have entered the uterine sinuses when thrown in from the cord, and, vice vers $\hat{a}$, that colouring matter has passed into the placental tufts when introduced by the maternal vessels : yet both these phenomena are fallacious, and due to very simple causes.

1. The umbilical arteries, after dividing and passing in a convoluted and serpentine form over the fotal surface of the placenta, dip at various intervals into its substance, there dividing and subdividing infinitely. The trunks are covered on the surface of the organ by the foetal membranes, and each branch, as it dips into the thickness of the tissue, carries before it a fold of the chorion.

2. The whole mass of the placenta is made up of the innumerable ramifications of the arteries, terminating in beautiful coiled and convoluted capillaries, (Pl. I. fig. 1.) which form tufts or bouquets at various intervals; these finally become continuous with the minute origins of the umbilical vein, which returns to the fotus in the same direction that the arteries left it, viz., coiled and twisted in the umbilical cord. 
3. The vein and its branches are greatly larger than the arteries and their subdivisions, but less numerous.

4. The direction of the branches of the umbilical arteries is from the fotal to the uterine surface of the placenta, passing obliquely from the centre to the circumference and edges. The veins return in the reverse direction. All the vessels, besides their own proper coats, are enclosed in a fold of chorion. (Pl. I. fig. 3.)

5. As the minute branches of the arteries terminate in serpentine and very intricately coiled capillaries, so are these latter subdivided into masses, or tufted and bouquet-like processes, clothed by prolongations of the before-mentioned membrane. (Fig. 2.)

6. This membrane (chorion) constitutes (by division into processes) true villi, and each villus contains a tortuous capillary, which entering from the arterial side leaves it by the venous : as the vessel leaves the villus there is a slight but manifest increase of size.

7. A single tuft or collection of villi, well injected, and laid flat under an inch, or half inch, objectglass, appears at first sight an inextricable confusion of curiously-contorted capillary vessels; but separated by needles, and a single villus detached, or expanded beneath a higher magnifying power, this seeming confusion is reduced to order, and the true anatomy of these vessels explained. (Fig. 2.)

8. The membrane enclosing the vessels and capil- 
laries is studded on the exterior by nucleated cells, resembling an irregular epithelium. (Fig. 3. c c.)

9. The enclosed tufts, or capillaries, nowhere anastomose with other than foetal or umbilical vessels.

10. The arteries and veins, though covered by a common membrane, are nowhere so closely bound together, as to constitute one undivided though really double vessel, as described by Dr. Reid,* and " the blunt extremities," adverted to by that gentleman, appear to me to be the villi of the placenta.

11. The villi are not connected together by cellular tissue, but the mass of the placenta is made up by the vascular divisions and subdivisions, and by the tufts or bouquets of capillaries; the interstices are everywhere free, and communicate with each other.

12. There are no distinct or defined cells constituting a maternal portion of the placenta.

13. The uterine surface of the organ is covered by the decidua, which does not appear to enter further than between the lobules, and the depth to which it thus penetrates varies with the extent of the fissures.

14. Stretching from the foetal to the uterine surface of the placenta are irregular semi-fibrous bands, more firm towards the foetal surface, and nearly disappearing towards the decidual: these appear to

* January number of Edinburgh Medical and Surgical Journal, 1841. 
give firmness to the spongy mass, and to a certain extent support to the tufts of villi.

15. The bouquets of capillaries are found in all parts of the placenta, but are more numerous at the uterine surface, where they will be found close beneath thordecidua.

16. Upon the decidual surface may be observed, thinly scattered, certain papillæ, somewhat obtuse and blunted, about a line and a half in length, which seem to be constituted by innumerable coiled and minute capillaries. Are these the analogues of the fœtal cotyledons?

On comparing these observations and the accompanying drawings, we cannot help being struck with the very strong resemblance they bear to the plates given in Wagner's Physiology as copied from Weber. Dr. Reid denies the correctness of Weber's drawings, as represented in the Icones Physiologicæ of Wagner. (Tab. xi. fig. 2.)

Nevertheless, in the plate appended to the present paper, which was made from drawings of portions of a placenta fully injected, but without extravation, the resemblance is so striking as to go a great way to prove the correctness of both draughtsmen, especially as they were made independent of a previous view of Wagner's Icones. One of the principal causes of difference that seems, to exist in the representations of the same part by different anatomists depends probably upon the different conditions of the injection. Where the vessels have not been fully filled, the extreme branches will present very diverse forms, and the degree of convolution of these minute tubes 
mainly depends upon their being accurately filled. Another cause of variation is the quantity of magnifying power used by the observer. It is true, the vessels described in this paper are well seen with even a two-inch object-glass, but it requires a far higher power to develop the real condition and arrangement of these structures. They should be viewed both as opaque and transparent objects, and it is not until the one eighth or perhaps one fourth of an inch object-glass is brought to bear, by transmitted light, upon the villi, that the true nature of the investing membrane is made apparent.

It has been observed by some anatomists, that the uterine veins may be filled by injection thrown in by the umbilical arteries. The explanation of this phenomenon is sufficiently easy. The tufted villi are very delicate, and it not unfrequently happens that the injection bursts through the covering of chorion, and so escapes into the interstices between the villi, which have been usually, but improperly, called the cells of the placenta. If the injection so escapes, it will easily find its way, after distending the spongy mass, into the uterine sinuses, and thus fill the uterine veins.

On the other hand, coloured fluid, thrown into either the uterine arteries or veins, will distend the placental or spongy interspaces, and if the foetal tufts be lacerated by the distension or force of the manipulation, some of it will enter the broken extremities of the fotal vessels. This, however, can never produce a perfect injection of the placenta, as 
the compression of the tufts from the injection around, and on the outside of them, will, except in very rare instances, prevent such a display.

If the maternal blood is extravasated into the spongy mass of the placenta by the " curling arteries," as supposed by John Hunter, or enters it in any other fashion, then the fotal tufts become, in function, absorbent villi ; they take up the necessary or nutrient part of that fluid, which is then carried to the fotus by the umbilical vein. The blood having circulated through the fotus, is in part returned to the placenta by the umbilical arteries: part however is retained, and appropriated to the nourishment and growth of the embryo. Thus the arteries, though two in number, are in aggregate diameter less than the vein which brings to the foetus the nutrient fluids of the mother.

In the placenta must go on a double action, or interchange of fluids; for the blood returned to this organ by the arteries is unfitted for a second circulation through the embryo: at least this is true in part if not entirely. Hence, while the blood, or nutrient material of the blood, brought by the uterine arteries, and previously aërated by the mother, enters by endosmose the absorbent capillaries of the fœtal villi, that portion of the fotal blood that requires the action of oxygen escapes by exosmose, and returns by the uterine sinuses and veins to the maternal heart. Thus the lungs of the mother are in fact the lungs of the foetus, and hence the comparatively simple system of the vessels of the cord. 
In the incubated hen's egg, taking that as the type of a different condition, we find separate systems for the accomplishment of the different objects.

The nutrient materials out of which the embryo has to be built up, are contained within the shell, and whatever may be the mysterious chemico-vital action between the albumen and the yolk, as developed by a definite temperature, yet it is very certain that the exquisitely beautiful arrangement of the vascular folds of the vitelline sac, heretofore called " vasa lutea," do absorb the nutrient fluids, out of which the blood of the young bird is elaborated. As, however, there is no provision for the aëration of the blood, in this vitelline sac, we find a new membrane, the allantois, developed, whose function, from the arrangement and subsequent termination of its vessels, cannot be doubted to be pulmonary.

Thus then we have a more complicated system of vessels than in the human cord; for besides the umbilical arteries and vein which spread out upon the allantois, we have the encephalo-mesenteric arteries and veins, which subserve the purpose of absorbing the nutrient fluids of the vitellicle.

In the human embryo a more simple mechanism performs the same necessities, in consequence of the intermediate connection of the fotus with the mother; while the vascular tufts remain the analogues of the vasa lutea and vitelline folds of the incubated egg. 


\section{Explanation of Plate I.}

Fig. 1. represents the arrangement of the umbilical vessels of the placenta, taken from a wet, injected preparation, and viewed with a power of 120 linear. A A, umbilical arteries ; B, umbilical vein ; c, coiled and convoluted capillaries.

Fig. 2. represents a tuft of villi of the placenta. A, chorion ; B, umbilical vessels ; c, capillary vessels of the villi; magnified 300 times linear.

Fig. 3. Two villi of the chorion united, enclosing the capillary termination of the artery, returning by the vein. A A, umbilical arteries; B, umbilical vein ; $\mathbf{c}$, chorion; $\mathrm{D}$, nucleated corpuscules in the chorion. 


$$
\therefore \quad \text { Vol.XXV. Pl.1. }
$$

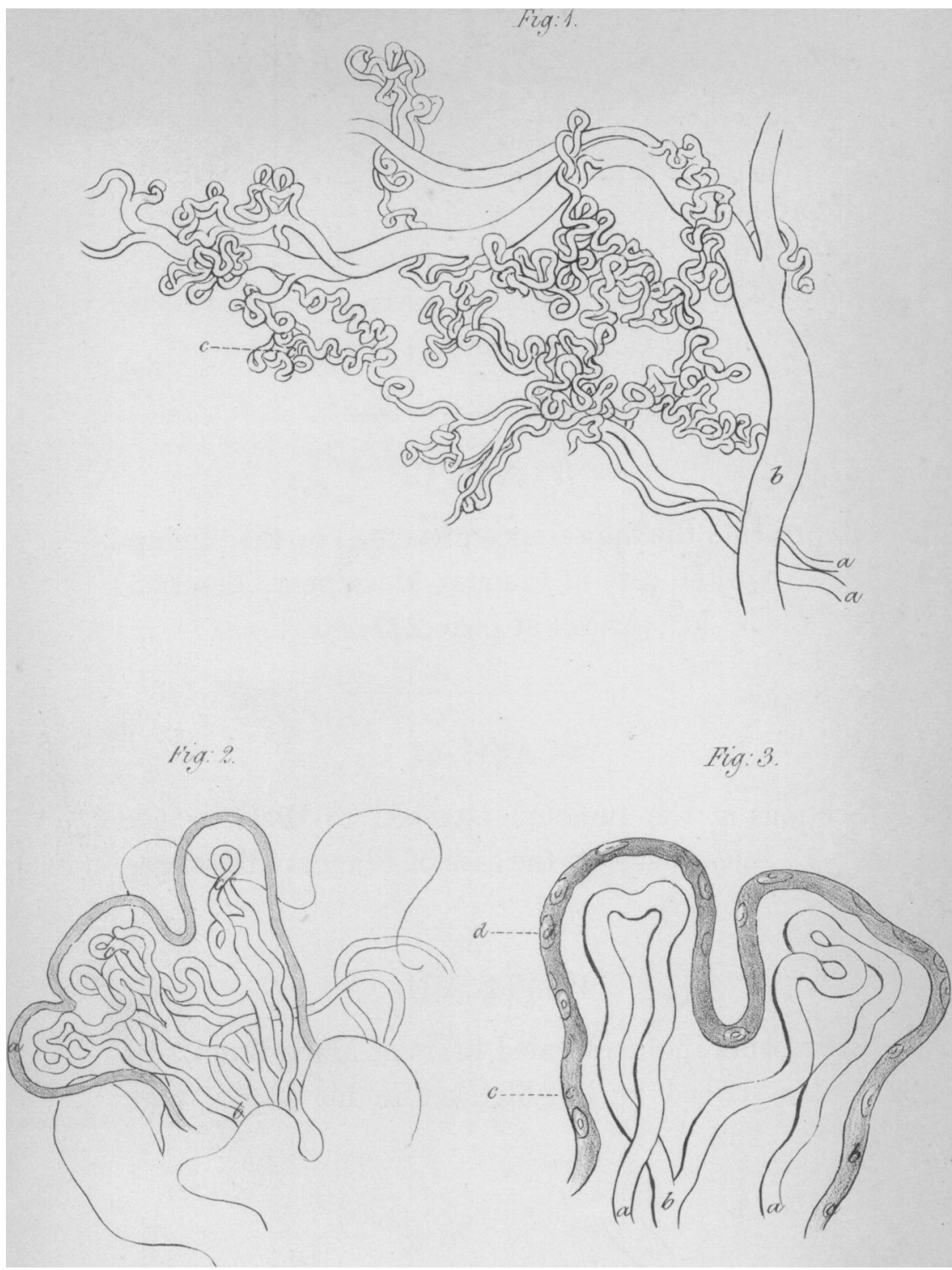

